www.jmscr.igmpublication.org

Impact Factor (SJIF): 6.379

Index Copernicus Value: 71.58

ISSN (e)-2347-176x ISSN (p) 2455-0450

crossref DOI: _https://dx.doi.org/10.18535/jmscr/v6i2.198

Journal Of Medical Science And Clinical Research

IGM Publication

An Official Publication of IGM Publication

\title{
Prospective Study of Two Management Strategies for Dirty Abdominal Incisions following Laparotomy for Ruptured Appendicitis
}

\author{
Authors \\ Idris Olawale Lateef, Idris Oluwatoyin Jelilat*, Adejumobi Musibau Olaniyi, \\ Kolawole Oladapo Adedayo, Akanbi Olusola Olateju, Oyeniyi Ganiyu Adebukola, \\ Oguntola Adetunji Saliu \\ Department of Surgery, Ladoke Akintola University of Technology Teaching Hospital, Osogbo, Osun State, \\ Nigeria \\ *Department of Medical Microbiology and Parasitology, College of Health Sciences, Ladoke Akintola \\ University of Technology, Osogbo. Osun State, Nigeria. \\ Corresponding Author \\ IDRIS Oluwatoyin Jelilat \\ Department of Medical Microbiology and Parasitology, College of Health Sciences, Ladoke Akintola \\ University of Technology, Osogbo. Osun State, Nigeria. \\ Telephone No; +2348033812425, Email: ojidris@lautech.edu.ng
}

\begin{abstract}
Background: The rate of surgical site infection (SSI) for dirty abdominal wounds is about 40\%. The optimal closure method, for dirty abdominal incisions, that will reduce SSI still remains controversial. The aim of this study is to determine the optimal method of wound closure, after laparotomy, for patients with ruptured appendicitis.

Methods: Eighty-seven patients were randomized into one of the primary closure $(P C)$ group and delayed primary closure (DPC) group. Patients in the PC group had their surgical incisions closed immediately while for those in the latter group had DPC. Incisions in the DPC group were evaluated on postoperative day-4 (POD4), for closure if pristine. However, if SSI was suspected in both groups, the dressing was removed for wound inspection using sterile technique (also in the PC group, sutures were removed over the infected area to allow egress of exudates), and wound dressing was commenced. The main outcome measures were the incidence of surgical site infection and the length of hospital stay.

Results: In the entire series, SSI developed in $40.2 \%$ of patients after closure of incisions. Primary closure group had a higher incidence of SSI (41.9\% vs 38.6\%, $p=0.088)$ and longer LOS $(8.1 \pm 3.7$ days vs $7.3 \pm 2.2$ days, $p=0.12$ ).

Conclusion: There was no significant difference in the development of SSI between the two groups ( $P$ $>0.05$ ). Laparotomy incisions, after ruptured appendicitis, most often can be closed primarily without a significant increase in the incidence of SSI as compared to DPC.

Keywords: PC, DPC, Ruptured appendicitis, SSI.
\end{abstract}




\section{Introduction}

Superficial incisional surgical site infection (superficial incisional SSI), previously known as postoperative wound infection, ${ }^{1,2}$ is the most common nosocomial infection in surgical patients, constituting about $40 \%$ of nosocomial infections, and is associated with substantial morbidity, undue expenses and mortality. ${ }^{1,2}$

The Centers for Disease Control and prevention (CDC) defines surgical site infection (SSI) as an infection that occurs at the incision site or organ / space explored, within thirty days after surgery or within one year if a prosthetic implant is put in place. $^{1,2}$ It is divided into incisional and organ / space infections. Incisional infection is further subdivided into superficial (involving the skin and subcutaneous tissues) and deep which involves the muscle and enveloping fascial layers. ${ }^{1-4}$

Surgical wound care is aimed at wound healing without complications, but dirty laparotomy wounds, resulting from causes such as ruptured appendicitis or typhoid perforation, are associated with high risk of SSI - the leading complication of peritonitis. ${ }^{1-4}$

The epidemiology of superficial incisional SSI has changed over the years as surgeons have learned to control bacteria and the inoculum because many components of the bacterial contribution to SSI are now clearly understood and measures to control bacteria are being implemented. ${ }^{4,5}$ The host factors have now become apparent, hence the increasing focus on the patient (the host) for measures that will continue to provide improved results. ${ }^{4,5}$

The method of wound closure has been known to influence the occurrence of superficial SSI. ${ }^{1-5}$ Two options exist for closure of dirty abdominal wounds; Primary wound closure (PC) and Delayed-primary wound closure (DPC). ${ }^{1-4}$ However, opinions on the optimal method of closure of dirty wounds are divided ${ }^{1-9}$.

Surgical treatment of a surgical site infection depends on the depth of infection; for superficial incisional SSI, sutures are removed over the area of infection to allow for drainage and regular wound dressing will be performed. Gentle exploration to break locules, saline irrigation and later, secondary closure may be necessary. ${ }^{2,6,10,11}$ However, for deep incisional SSI with pus coming from beneath the fascia, intra-abdominal abscess should be considered and this can be drained by possible reoperation especially when loculated or percutaneously under ultrasound/ computed tomography (CT)-guidance., ${ }^{4,5}$

When superficial SSI is assessed prospectively and accurately, it is observed to affect more patients than previously thought. ${ }^{12}$ Finding a reliable wound closure method to reduce SSI following laparotomy for secondary peritonitis is an international research priority because of its attendant significant morbidity, mortality and financial cost. ${ }^{12,13}$

In a bid to finding answers to this, we thus conducted a prospective randomized study with the aim of comparing the incidence of superficial SSIs between DPC and PC of laparotomy wounds, in patients with ruptured appendicitis.

\section{Methodology}

This study was a prospective randomized study involving patients with peritonitis secondary to ruptured appendicitis, who were operated on between June, 2009 and June, 2015. Ruptured appendicitis (in patients with generalized peritonitis) was defined as gross rupture of the inflamed appendix identified by the surgeon at the time of operation. This study was ethically approved by Ethics and Research Committee of the institution and written informed consent was obtained from each patient before being enrolled into the study. We recorded patient-related factors that could have contributed to SSI: Age, gender, duration of symptoms, packed cell volume (PCV), body mass index (BMI), serum protein and albumin, serum electrolyte urea and creatinine, jaundice, comorbid illnesses (diabetes mellitus, malignancy, chronic streroid therapy, chemotherapy and HIV-AIDS). The operation related factors recorded were type of anaesthesia, type and length of incision, intraoperative 
findings, method of closure of the incision (skin and subcutaneous layer), duration of surgery, postoperative complications and length of hospital stay were also recorded. The inclusion criteria were patients who are 16 years or older, either gender, with gross rupture of inflamed appendix. Exclusion criteria were patients younger than 16years, with immunosuppressive conditions like the human immunodeficiency virus (HIV) infection with acquired immunodeficiency syndrome (AIDS), jaundice, diabetes mellitus, obesity, subcutaneous layer thickness of more than $2 \mathrm{~cm}$, malignancy, patients on steroids and chemotherapy as well as all cases of microscopic perforation of inflamed appendix identified by the pathologist and death of patients before postoperative day 30. All patients received perioperative intravenous ciprofloxacin and metronidazole and these were adjusted according to the response of the patients and results of wound swab microscopy $(\mathrm{M} / \mathrm{C} / \mathrm{S})$. They all had appendectomy though a midline infraumbilical incision. Peritoneal lavage was done with warm $0.9 \%$ saline until clear effluent was returned. Soft latex silicon coated 20FR 2-way Foley catheter was used as drain placed in the pelvis through a separate incision on the anterior abdominal wall. Mass closure of the peritoneum and fascia was done with a non-absorbable, monofilament number one nylon sutures. Finally, the incision (skin and subcutaneous tissue) was either closed primarily or left open for delayed primary closure, according to randomization. Incisions were closed in either technique with a non-absorbable monofilament number $2 / 0$ nylon suture material.

Patients in the primary closure (PC) group had their surgical incisions (skin and subcutaneous tissue) closed immediately while in delayed primary closure (DPC) group, the incisions were left open (packed with $0.9 \%$ saline soaked gauze) and were not manipulated until postoperative day3 , for inspection and closure if pristine. However, if SSI was suspected in both PC and DPC groups based on local features like pain accompanied by serous or purulent discharge from the wound, foul odour of the wound or systemic signs (fever, tachycardia) before the scheduled first dressing or during serial follow up, the dressing was removed for wound inspection using sterile technique (also in the PC group, sutures were removed over the area of swelling and redness to allow for drainage of exudates), wound swabbing for microscopy, culture and sensitivity (MCS) was done and wound dressing was commenced and continued until the wound was clean enough for secondary closure. All patients were followed up for one month after surgery.

The sample size was estimated to be 42 for each group in comparison using modified Kirkwood formula ${ }^{14,15}$ and sampling procedure was simple randomization, by alternating technique. ${ }^{7,16}$ The data was recorded on a pre-designed proforma and subsequently entered into Statistical Package for Social Science - SPSS version 20.0 which was used for the statistical analysis..

\section{Results}

A total of 92 patients were randomized. Three patients ( 1 in PC group and 2 in the DPC group) were withdrawn because of death before postoperative day (POD)-30, and two in the PC group were lost to follow up before POD-30. The remaining 87 patients (43 in the PC group and 44 in the DPC group) received the allocated intervention. Both groups of patients were similar in terms of age, aetiology of dirty wound and risk factors for SSI. There was no significant difference in the duration of symptoms before presentation $(\mathrm{p}=0.273)$. There were significantly more men in the PC group (32 vs. $11, \mathrm{P}=0.025$, chi-square; (Table 1) 
Table 1: Patient demographics and clinical detail

\begin{tabular}{lccc}
\hline & $\mathrm{DPC}(n=44)$ & $\mathrm{PC}(n=43)$ & $p$ value \\
\hline Male / female & $22 / 22$ & $32 / 11$ & 0.019 \\
Mean age (years) & $27.07 \pm 6.3$ & $29.14 \pm 11.9$ & 0.313 \\
Risk factors & & & \\
ASA class (IIE/ IIIE) & $39 / 5$ & $41 / 2$ & 0.028 \\
BMI (<18/ 18-30) & $2 / 42$ & $14 / 29$ & 0.169 \\
Serum Alb (g/dl) [<3/3-5] & $2 / 42$ & $5 / 38$ & 0.002 \\
Mean DOS (Hours) & $82.36 \pm 1.6$ & $87.07 \pm 1.8$ & 0.618 \\
Duration of Op (Hours) [<2/ $\geq 2] 44 / 0$ & $41 / 2$ & 0.024 \\
\end{tabular}

Data are presented as mean \pm standard deviation or $\mathrm{n}(\%)$.

Abbreviations: DPC $=$ delayed primary closure, $\mathrm{PC}=$ primary closure, ASA class $=$ American Society of Anaesthesiologists classification, $\mathrm{BMI}=$ body mass index, Alb = albumin, DOS - duration of surgery, Op = operation

In the entire series, surgical site infection developed in $40.2 \%$ of patients after closure of incisions. The PC group had a higher incidence of SSI $(41.9 \%$ vs $38.6 \%, \mathrm{p}=0.088)$ and longer LOS $(8.1 \pm 3.7$ days vs $7.3 \pm 2.2$ days, $\mathrm{p}=0.12)$. All patients were followed up till POD-30.

The bacterial cultured from infected incisions were similar in both groups with a preponderance

Table 2. Bacteria cultured from wound swab $(n=35)$ of gram negative bacteria. The most common organisms cultured from the wounds were Klebsiella species (32.1\%), Escherichia coli (25.0\%), Staphylococcus aureus (21.5\%) and Streptococci species (14.3\%). About $7.1 \%$ of bacterial culture were polymicrobial (E. coli, Pseudomonas spp, Klebsiella); (These results are shown in Table 2).

\begin{tabular}{|l|c|c|c|c|}
\hline Culture yield & DPC & PC & Frequency & Percentage \\
\hline No growth & 7 & 0 & 7 & 20.0 \\
Escherichia coli & 2 & 5 & 7 & 20.0 \\
Streptococcal species & 1 & 3 & 4 & 11.4 \\
Klebsiella species & 5 & 4 & 9 & 25.7 \\
Staphylococcus aureus & 0 & 6 & 6 & 17.2 \\
Polymicrobial & 2 & 0 & 2 & 5.7 \\
Total & 17 & 18 & 35 & 100.0 \\
\hline
\end{tabular}

Abbreviations: DPC - delayed primary closure, PC - primary closure.

All of the 7 patients with ASA IIIE class developed superficial SSI with $2 / 43(4.65 \%)$ in the PC group and 5/44 (11.36\%) in the DPC group ( $\mathrm{p}=0.144$ vs 0.009 respectively).

There was no significant difference in the rate of SSI and duration of operation in the PC and DPC group ( $\mathrm{p}=0.502)$.

In the PC group, $38(88.4 \%)$ patients had normal preoperative serum albumin values of $3-5 \mathrm{~g} / \mathrm{dl}$ out of which $36.8 \%$ (14/43) developed superficial SSI however $25 \%(1 / 4)$ of patients with hypoalbuminaemia developed superficial SSI.

In the DPC group, 42 (95.5\%) patients had normal preoperative serum albumin values, out of which $35.7 \%$ (15/42) developed superficial SSI but all patients (2/2) with hypoalbuminaemia in this group developed superficial SSI. ( $\mathrm{p}$ values: $\mathrm{PC}=$ 0.88 and $\mathrm{DPC}=0.144$ ).

The length of hospital stay was longer in the PC group but not statistically significant, $(8.1 \pm 3.7$ days vs $7.3 \pm 2.2$ days, $\mathrm{p}=0.12$ ).

\section{Discussion}

The incidence of superficial incisional SSI has changed over time as surgeons have learned to control bacteria and the inoculum because many components of the bacterial contribution to superficial incisional SSI are now clearly understood and measures to control bacteria are being implemented. ${ }^{5,6}$ The host factors have now 
become apparent, hence the increasing focus on the patient (the host) for measures that will continue to provide improved results. ${ }^{5,6}$ The method of wound closure, Primary wound closure and Delayed-primary wound closure, has been known to influence the occurrence of superficial SSI. ${ }^{1-10}$. However, opinions on the optimal method of closure of dirty wounds are divided..$^{1-4,7-10}$

Delayed primary skin closure was accepted as the optimal method to treat wounds of war. ${ }^{1-8}$ Complex soft tissue injuries sustained during combat are still debrided and closed in a staged fashion (although the dressings used in the interim might have changed). ${ }^{1-8}$ The simplicity and effectiveness of this wound care policy led some to extrapolate the technique to contaminated and dirty laparotomy wounds, such as those resulting from ruptured appendicitis.

Proponents of PC have however cited better patient acceptance, minimal discomfort, easy postoperative incision management, shorter hospitalization, and the low clinical and financial implication of incisional infections as some of the reasons. $^{1-4,7-10}$

Our study demonstrates that PC of laparotomy incisions, after ruptured appendicitis, most often can be performed without a significant increase in the incidence of SSI as compared to DPC.

Several other trials have also reported no significant difference in SSIs rates between PC and DPC of laparotomy incisions after ruptured appendicitis.

Our study as well as Tsang et $\mathrm{al}^{17}$ and Lemiew et $\mathrm{al}^{18}$ used normal saline as the gauze-soaking agent in the DPC group whereas Pettigrew et $\mathrm{al}^{19}$ and Mc Greal et $\mathrm{al}^{20}$ used Bethadine solution as the soaking agent with no difference in the SSIs rate in the DPC groups between the two methods of wound packing while Boonying et $\mathrm{al}^{4}$ reported lower superficial SSIs rate in PC group than in DPC group. Table 3, summarized the results of these previous prospective randomized trials comparing DPC with PC of laparotomy incisions following Ruptured or gangrenous appendicitis.

In this study all patients with ASA class III had superficial SSI which further underscored the influence of ASA score on the frequencies of SSI. This is similar to what was reported by Mansoor khan etal ${ }^{21}$, that ASA scoring system has definite influence on the frequency of surgical site infections and that the SSI rates increase with the increasing ASA scores.

Table 3. Results of studies of DPC vs. PC following laparotomy for Ruptured appendicitis

\begin{tabular}{|c|c|c|c|c|c|c|}
\hline \multirow[b]{2}{*}{ Authors } & \multirow[b]{2}{*}{ Type of wound } & \multicolumn{2}{|c|}{ DPC } & \multicolumn{2}{|r|}{$\mathrm{PC}$} & \multirow[b]{2}{*}{$\begin{array}{l}\text { Comments on difference } \\
\text { in infection rate }\end{array}$} \\
\hline & & $\mathrm{n}$ & SSI $(\%)$ & $\mathrm{n}$ & SSI $(\%)$ & \\
\hline Grosfeld (1968)16 & Post R.Ap & 44 & $1(2.3 \%)$ & 41 & $14(34.1 \%)$ & DPC is better (significant) \\
\hline Andersen (1972)18 & Post R.Ap & 58 & $15(26 \%)$ & 58 & $20(34 \%)$ & Not significant \\
\hline Pettigrew (1981)27 & Gangrenous \& R.Ap & 42 & $23(54 \%)$ & 41 & $15(37 \%)$ & Not significant \\
\hline Tsang et al. (1992)28 & R.Ap. \&Gangrenous Ap & 25 & $6(24 \%)$ & 38 & $8(21 \%)$ & Not significant \\
\hline Lemieur (1999)25 & Post R.Ap. & 27 & $0(0 \%)$ & 29 & $7(24 \%)$ & Significance not mentioned \\
\hline Rucinski (2000) & Post R.Ap & 808 & $42(4.6 \%)$ & 1724 & $91(4.7 \%)$ & Not significant \\
\hline Cohn (2001) & Post R. Ap & 9 & $5(55.6)$ & 8 & $4(50 \%)$ & Not significant \\
\hline Mc Greal (2002) & Post R. Ap & 34 & $8(23.5 \%)$ & 26 & $2(7.7 \%)$ & $\mathrm{PC}$ is better (significant) \\
\hline Chiang (2012) & R.Ap.\& Gangrenous Ap & 34 & $1(2.9 \%)$ & 36 & $14(38.9)$ & DPC is better (significant) \\
\hline Yousaf (2014) & R.Ap.\& Gangrenous Ap & 55 & $11(20 \%)$ & 55 & $5(09 \%)$ & Not significant \\
\hline Current study & Post R. Ap & 44 & $17(38.6 \%)$ & 43 & $18(41.9 \%)$ & Not significant \\
\hline
\end{tabular}

Abbreviations: DPC $=$ delayed primary closure, $\mathrm{PC}=$ primary closure, $\mathrm{R} . \mathrm{Ap}=$ Ruptured appendicitis, Ap $=\mathrm{Appendicitis}$. 
Other than the National Research Council (NRC) wound classification according to the risk of SSI (i.e., clean, clean-contaminated, contaminated, and dirty), some other risk factors like duration of operation and ASA classification have been validated and included in risk classification scores by National Nosocomial Infections Surveillance (NNIS) for better prediction of postoperative superficial SSI. ${ }^{5,6}$

However, more accurate risk classification scores, by including all possible risk factors in NNIS criteria as well as host defense mechanism (immunosuppression / immunosuppressive agents, co-morbidities like Diabetes mellitus, subcutaneous fat thickness etc.), can help a physician to more accurately estimate the probability of postoperative superficial SSI as his will influence the physician's judgment on whether to apply PC or DPC, thus lead to better wound management decisions.

Tsang $e$ t $a l^{17}$ studied patients with gangrenous or ruptured appendicitis and found no difference in the rate of SSI between the two groups. Pettigrew ${ }^{19}$ and Andersen et al ${ }^{22}$ both randomized more than 100 patients each with gangrenous or perforated appendicitis to DPC group versus PC group. These authors used topical antibiotics in one or more randomized arms, and in spite of this, they found no significant difference SSI rate in the two groups. ${ }^{11,17}$.

However, these studies were at least 20 years old, highlighting the need to address this issue with a more recent trial ${ }^{11}$

\section{Conclusion}

Our study suggested that the incidence of superficial SSI in ruptured appendicitis as well as the length of hospital stay (LOS) were not different between primary and delayed primary closure groups. However, primary closure of laparotomy incisions, following ruptured appendicitis, may represent a simple, reliable and potentially cost-effective way of managing such wounds. A well designed, large-numbered multicenter randomized controlled trial would be warranted.

\section{References}

1. Jadesh Bhadragoudra, Basavaraj Narasanagi, Tejaswini Vallabha and Vikram Sindagikar; Comparative study of delayed primary closure versus primary closure of skin in contaminated and dirty abdominal wounds/ incisions. Int $\mathrm{J}$ Clin and Biomed Res. 2016;2(1): 16-19

2. Cohn SM, Giovanni Giannotti, Adrian W, Varela JE, Shartz DV, McKenney MG, Danny S, Enrique G, Jeffrey S, Patricia M, Laurence RS, Michael DH and Nicholas N.; Prospective randomized trial of two wounds management strategies for dirty abdominal wounds in Annal of Surgery, March 2001: 233 (3): $409-413$

3. Ruey-An Chiang, Shan-Long Chen and YaoChung Tsai; Delayed primary closure versus primary closure for wound management in perforated appendicitis: A prospective randomized controlled trial. Journal of Chinese Medical Association 75 (2012) 156 159

4. Boonying Siribumrungwong, Kanoklada Srikuea and Ammarin Thakkintian; Comparison of superficial surgical site infection between delayed primary and primary wound closures in ruptured appendicitis. Asian Journal of Surgery (2014) 37, $120-124$.

5. Mahmoud N. Kaylat and Merril T Dayton, Surgical complications in Sabiston textbook of Surgery; $18^{\text {th }}$ Edition, Courtney MT, Daniel RB, Mark ME, and Kenneth LM,(editors): Elsevier Saunders; 2007: 299305.

6. Jonathan L., Meakins, and Byron J. M., Prevention of postoperative infections in American College of Surgeons (ACS) Principle and practice of surgery, 2005: chapter 2: 1-19. 
7. Dipesh D, Duttaroy JJ, Bithika D, Ujjwal Bansal, and Prarthna D, Management Strategy for Dirty Abdominal Incisions: Primary or Delayed Primary Closure? A Randomized trial in Surgical infection in Ann Surgery, April 2009, 10:2: 129 - 136. [HINARI].

8. Adesunkanmi ARK and Ajao OG, Tyhoid ileal perforation; the value of delayedprimary closure of abdominal wounds in African Journal of Medical Science; 1996: 25: $31-35$.

9. Christopher OB, Primary versus Delayed primary closure of dirty abdominal wounds in National Postgraduate Medical College of Nigeria (NPMCN) Library; Nov 1991: 84 90.

10. Felipe AR, Enrique L, Roberto S and Patricia OS, Open versus Closed mana-gement of the abdomen in surgical treatm-ent of severe secondary peritonitis: A randomised clinical trial in British Journal of Surgery; 2007: 9 (11): $1317-1318$.

11. Mukhtar Ahmad, Kishwar Ali, Humera Latif, Saman Naz and Khalid Said; Comparison of primary wound closure with delayed primary wound closure in perforated appendicitis. $\mathrm{J}$ Ayub Med Coll Abbottabad, 2014; 26 (2)

12. Aneel Bhangu, Prashant Singh, Jonathan Lundy and Douglas M. Bowley; Systemic Review and Meta-analysis of Randomized Clinical Trials Comparing Primary vs Delayed primary Skin Closure in Contaminated and Dirty Abdominal Incisions. JAMA Surgery, August 2013, Volume 148, Number 8

13. Johnstone JMS, and Rintoul RF, Abdominal surgery; access and procedures in Farquharson's textbook of operative surgery; $8^{\text {th }}$ edition, RF Rintoul (editor), Churchill Livingstone; 2000: 337 - 352.

14. Avril Drumond, The research process in Research methods for therapists, $3^{\text {rd }}$ edition, Campling JO (editor), Stanley Thornes (Publishers) Ltd; 1998: 31 - 46.
15. Afolabi EB, Determination of sample size in a companion of medical statistics. $1^{\text {st }}$ Edition, Ibipress \&Publishing Co; 2006: 78- 156.

16. E.V. Ussiri, C.A. Mkony and M.R. Aziz; Sutured and Open Clean-Contaminated and Contaminated Laparotomy Wounds at Muhimbili National Hospital: A Comparison of Complications. East and Central African Journal of Surgery, Volume 9, Number 2 December 2004.

17. Tsang TM, Tam PKH and Saing H. Delayed primary wound closure using skin tapes for advanced appendicitis in children. Arch Surg 1992;127:451-53.

18. Lemieur TP, Rodriguez JL, Jacobs DM, Bennett ME and West MA. Wound management in perforated appendicitis. Am Surg 1999;65: 439-43.

19. Pettigrew RA. Delayed primary wound closure in gangrenous and perforated appendicitis. Br J Surg 1981;68:635-8.

20. McGreal GT, Joy A, Manning B, Kelly JL, O'Donnell JA, Kirwan WW and Redmond HP; Antiseptic wick: does it reduce the incidence of wound infection following appendectomy? World J Surg. 2002;26(5):631-634.

21. Mansoor Khan, Rooh-ul-Muqim, Mohammad Zarin, Jawad Khali and Muhammad Salman; Influence of ASA Score and Charlson Comorbidity Index on Surgical Site Infection Rates. Journal of College of Physician and Surgeon 2010, Vol.20 (8):506-509

22. Andersen B, Bendtsen A, Holbraad L, and Schantz A; Wound infections after appendicectomy. I. A controlled trial on the prophylactic efficacy of topical ampicillin in non-perforated appendicitis. II. A controlled trial on the prophylactic efficacy of delayed primary suture and topical ampicillin in perforated appendicitis. Acta Chir Scand 1972;138:531-6. 